\title{
Rapid astroglial stress response and elevation of endogenous BiP levels promote neuronal survival in hippocampal slice cultures
}

Andrea Gühna 1, Jasmin Traichel 2, Lepu Zhou 3, Ingrid Haas 4, Adrienne W Paton 5, James C Paton 5, Bernd Heimrich ${ }^{2,7}$ and Felicitas Pröls 6, ${ }^{*}$

${ }^{1}$ Institute of Anatomy, Department of Molecular Embryology, Medical Faculty, University of Freiburg, Freiburg, Germany.

2 Department of Neuroanatomy, Institute of Anatomy and Cell Biology, Medical Faculty, University of Freiburg, Freiburg 79104, Germany.

${ }^{3}$ Institute of Neuroanatomical, University Medical Center Hamburg-Eppendorf, Hamburg, Germany.

${ }^{4}$ Max-Planck-Institute for Immunology and Epigenetics, Stübeweg 51, 79108 Freiburg, Germany.

${ }^{5}$ Research Centre for Infectious Diseases, Department of Molecular and Biomedical Science, University of Adelaide, Adelaide, South Australia, 5005, Australia.

${ }^{6}$ Institute of Anatomy II, University of Cologne, Faculty of Medicine, Cologne, Germany.

${ }^{7}$ Center of Basics in NeuroModulation (NeuroModulBasics), Medical Faculty, University of Freiburg, Freiburg, Germany.

World Journal of Biology Pharmacy and Health Sciences, 2021, 07(02), 019-031

Publication history: Received on 24 June 2021; revised on 27 July 2021; accepted on 29 July 2021

Article DOI: https://doi.org/10.30574/wjbphs.2021.7.2.0078

\begin{abstract}
A hallmark of neurodegenerative diseases is the accumulation of protein aggregates, the formation of which is prevented by chaperone proteins. BiP is the central chaperone in the endoplasmic reticulum. In this study we investigated the pattern of BiP in tunicamycin-stressed murine organotypic hippocampal slice cultures (OHCs). In stressed OHCs highest apoptotic rates occur in neurons of the CA1 regions and the dentate gyrus, in which we found BiP levels to be lowest. Highest BiP protein levels were found in astrocytes. Cell culture experiments indicated that the stress response of glial cells is faster and stronger than in neuronal cells. We hypothesize that the rapid and pronounced BiP expression in astrocytes helps to maintain the fine-balanced micromilieu necessary for survival of neurons. SubAB is a toxin, which cleaves and inactivates BiP. Low dosages of SubAB did not elicit a specific glial response and apoptosis was not induced in a specific hippocampal subfield. Mild prestressing with SubAB promoted neuronal viability in tunicamycin-treated OHCs. We conclude that preconditioning of hippocampal tissue with stressors that elevate endogenous chaperone levels exert a protective effect thereby promoting neuronal survival. These experiments strengthen the thesis that preconditoning with mild stressors positively affects the survival of neuronal cells.
\end{abstract}

Keywords: Chaperone; Organotypic hippocampal slice cultures; Neuronal survival; Astrocytes; Tunicamycin

\section{Introduction}

\subsection{Chaperones in the ER and UPR signaling with focus on BiP}

The neuronal microenvironment is controlled by the activity of the neuronal and glial network. The biochemical composition of the neuronal microenvironment changes due to (patho-) physiological stimuli, ionic imbalances, nutrient deprivation, or temperature shifts. Prerequisite for the maintenance of cellular function is a tight control of the endogenous protein homeostasis. Chaperones mediate and control the correct folding, transport as well as the degradation of almost every cellular protein. Proteins destined for their insertion into the plasma membrane or for

\footnotetext{
* Corresponding author: Felicitas Pröls

Institute of Anatomy II, University of Cologne, Faculty of Medicine, Cologne, Germany.
} 
secretion into the extracellular space have to transit the endoplasmic reticulum (ER), where their processing and folding is mediated and surveyed by the ER-specific chaperone machinery. In case of accumulation of misfolded proteins in the ER, a signaling pathway, the unfolded protein response (UPR), is induced which leads to an overall arrest of protein synthesis to limit further protein load of the ER. Simultaneously the synthesis of chaperones and membrane components of the ER is enhanced to increase its folding and degradation capacity [1]. To date, three major UPR signaling pathways are known, which are mediated by PERK, IRE1 $\alpha$ and ATF6, all three of them being receptors in the ER membrane. They are silenced by their binding to ER-resident chaperone BiP, also known as Grp78, which is located at the luminal side of the ER membrane. BiP, thus, plays a central role in controlling the initiation of UPR signaling cascades. The luminal domains of IRE1 $\alpha$ and PERK have similarities in their sequences and structure suggesting a mechanism of action for both IRE1 $\alpha$ and PERK [2]. PERK activation leads to autophosphorylation and results in an overall arrest of protein translation via phosphorylation of the translation initiation factor eIF2 $\alpha$ [3]. The activation of IRE1 $\alpha$ also leads to autophosphorylation at the cytosolic site by its intrinsic kinase activity resulting in stimulation of the endoribonuclease activity and the subsequent cleavage of Xbp1-mRNA into its spliced variant (Xbp1s). Translated Xbp1s functions as a transcription factor, which induces transcription of UPR target genes that increase the cells' capacity for protein folding and protein degradation [2]. Furthermore, activation of the endonuclease activity degrades mRNA at the ER membrane, thus helping to further reduce the protein load of the ER in a process called regulated IRE1 $\alpha$ dependent decay (RIDD) [4]. Activation of ATF6 results in its transit to the Golgi and the cleavage of its cytosolic domain, which subsequently translocates into the nucleus to upregulate transcription of chaperone genes to increase the folding capacity in the ER [5].

BiP exerts multiple functions in the cell. In the ER, BiP mediates the translocation of newly synthesized polypeptides across the ER membrane. It facilitates the folding and assembly of proteins, targets misfolded proteins to the degradation pathway (ERAD), regulates calcium homeostasis, serves as an ER stress sensor, and controls the activation of the UPR [6]. Besides all these functions of the ER-localized BiP, other cellular localizations have been described including the cell surface, cytosol, mitochondria, and the nucleus, where BiP possibly controls signaling, proliferation, invasion, apoptosis, inflammation, and immunity [7].

\subsection{Neurodegeneration and accumulation of protein aggregates}

Formation and deposition of protein aggregates are structural hallmarks associated with various neurodegenerative diseases, such as Parkinson's, Huntington's and Alzheimer's diseases (AD). ER stress, elicited by protein aggregates, is known to be a common determinant of these neurodegenerative diseases [1, 8]. In Alzheimer's disease, hyperphosphorylated cytoskeletal tau protein forms aggregates that are deposited intracellularly [9, 10]. Another feature in $\mathrm{AD}$ is the appearance of insoluble amyloid-rich plaques in the extracellular space. Their formation is based on cleavage of the transmembrane $\beta$-amyloid precursor protein (APP) by presenilin 1 and presenilin 2 giving rise to A $\beta 40$ and $A \beta 42$, two peptides that tend to self-assemble into soluble, prefibrillar oligomers eventually forming solid fibrils [11]. The small, prefibrillar, soluble oligomers are found extra- as well as intracellularly and are considered to be the most neurotoxic form [12]. Their neurotoxicity might be based on an increased chaperone demand for binding, solubilizing and renaturing the oligomers. The formation of oligomer-chaperone heteroprotein complexes might function as a chaperone sink resulting in a reduction of the free functional chaperone pool. This hypothesis is also strengthened the development of proteinopathies in aging brains, in which the level of functional chaperones is reduced, the adaptive UPR signaling decreased while the protein load is increased due to increased mutational rates [1, 13]. Overall, imbalances in protein to chaperone ratios induce the UPR in order to resume the folding capacity by a general translational arrest on the one hand and by the upregulation of chaperone levels on the other hand. Pathohistochemical staining of brains of AD patients revealed increased levels of phosphorylated PERK, eIF $2 \alpha$ and IRE1 $\alpha$ in hippocampal neurons associated with granulovacuolar degeneration $[14,15]$. The induction of the IRE1 $\alpha / \mathrm{Xbp} 1$ pathway seems to offer a neuroprotective gate, since overexpression of spliced Xbp1 enhanced $A \beta 42$ clearance and improved geotaxis, as was shown in aged flies [16]. Furthermore, it has been shown to improve hippocampal synaptic plasticity [17]. On the other hand, deletion of IRE1 $\alpha$ significantly reduced amyloid deposition and the content of $\beta$-amyloid oligomers and furthermore restored the learning and memory capacity in an AD mouse model [18]. A plausible explanation for the underlying mechanism of this dual function of the IRE1 $\alpha / \mathrm{Xbp} 1$ branch could be that the activation of Xbp1 is not solely triggered by phosphorylation of IRE1 $\alpha$ and that the beneficial or deleterious effect of the UPR is context-dependent and possibly controlled by signaling networks.

\section{Aim}

In order to investigate early molecular responses of neuronal cells when exposed to imbalances in protein to chaperone levels, we examined the BiP pattern and neuronal apoptosis after application of two different stressors in organotypic hippocampal slice cultures (OHCs). Tunicamycin is a glycosylation inhibitor, its application results in accumulation of 
unglycosylated, misfolded proteins. The toxin SubAB cleaves and inactivates BiP in an enzymatic manner [19]. We show that tunicamycin treatment upregulates BiP levels primarily in glial cells. In contrast, low BiP levels are found in neuronal cells, especially in the vulnerable hippocampal regions CA1 and the dentate gyrus. Treatment of the OHCs with SubAB did not elicit a specific glial stress response but induced apoptosis in a dose-dependent manner in all hippocampal regions. Preconditioning of OHCs with low doses of SubAB protected hippocampal neurons from subsequent tunicamycin-elicited stress, provided that SubAB dosages were as low as to allow the maintenance of endogenous BiP levels by compensatory upregulation of BiP.

\section{Material and methods}

\subsection{Organotypic slice cultures}

Hippocampi from neonate C57/Bl6 mice (P0-P2) were cut perpendicularly to their longitudinal axis into $400 \mu \mathrm{m}$ thick slices and cultured as described previously in control medium for $48 \mathrm{~h}$ [20]. SubAB treatment: OHCs were treated with SubAB at day 3 (days in vitro, DIV3) for 48 hours. At DIV5 toxin-containing medium was replaced by control medium and OHCs were incubated for another $48 \mathrm{~h}$. Tunicamycin treatment: OHCs were incubated in control medium for 5 days. At DIV5 tunicamycin (300 ng/ml) was added for another 48 hours prior to fixing or lysing the tissue. Preconditioning with SubAB (1 ng/ml) was performed at DIV3 for 48 hours followed by tunicamycin (300 ng/ml) treatment at DIV5 for additional 2 days. At DIV7 cultured hippocampi were either fixed with 4\% PFA in $0.1 \mathrm{M}$ PB for $2 \mathrm{~h}$ for subsequent immunohistochemical studies or lysed for RNA or protein isolation. All experiments were performed at least three times. All animal experiments were carried out in accordance with the EU Directive 2010/63/EU.

\subsection{Immunohistochemical staining}

For immunohistochemical staining, PFA fixed OHCs were rinsed in $0.1 \mathrm{M}$ (phosphate-buffer saline) PBS and cut into 50 $\mu \mathrm{m}$ vibratome sections. After tissue permeabilization with $0.3 \%$ Triton-X100 and blocking in $5 \%$ normal goat serum and 1\% BSA for $1 \mathrm{~h}$, slices were incubated in primary antibodies (rabbit anti-BiP/Grp78 1:1000 (Abcam ab21685, mouse anti-NeuN (1:1500 Abcam ab104224 diluted in 1\% BSA/PBS), mouse anti-GFAP (1:500, Sigma G3893) over night at $4^{\circ} \mathrm{C}$. Prior immunolabelling for cleaved caspase-3, sections were permeabilized with $0.1 \%$ Triton-X100 and $1 \%$ NGS in TBS (rabbit anti-cleaved Caspase-3 1:1500 (Cell Signalling \#9661). After washing, sections incubated with 2nd fluorescent antibody (Alexa fluor 488 (Molecular Probes), Cy3 (Jackson ImmunoResearch) diluted in 0.1\% BSA/PBS) for $2 \mathrm{~h}$ at room temperature. After careful and thorough washing of the sections, nuclei were stained by incubation in 300 nM DAPI solution (Hoechst 33258) for $10 \mathrm{~min}$. After washing, sections were mounted on uncoated slides, coverslipped using Immu-Mount and digitally photographed using a Zeiss Apotome microscope.

\subsection{Cell culture experiments}

Mouse Neuro2a cells (N2a, American Type Culture Collection) were cultured up to passage 10. Murine primary astrocytes (cultured up to passage 3) were kindly isolated and supplied by Björn Spittau (University of Freiburg) according to the protocol [21] and cultured no longer than 3 passages. Cells were cultured in DMEM, including high glucose, $200 \mu \mathrm{M}$ L-glutamine and $10 \% \mathrm{FCS}$, at $37^{\circ} \mathrm{C}$ in an incubator (5\% CO2) and treated with tunicamycin $(1 \mu \mathrm{g} / \mathrm{ml})$, thapsigargin $(1 \mu \mathrm{M})$ or SubAB $(1 \mu \mathrm{g} / \mathrm{ml})$ if not otherwise indicated. Cells were lysed and total RNA was isolated for subsequent PCR analyses (Qiagen). In order to avoid DNA contaminations care was taken to intensively treat the lysates with DNase as suggested by the protocol supplied by Qiagen. All experiments were performed three times in triplicate.

\subsection{Western Blot Analysis}

For protein extraction, cultured cells were carefully washed twice in PBS and lysed in M-Per buffer (Perbo Pierce Laboratories), supplemented with Complete, a protease inhibitor cocktail (Hoffmann-La-Roche), for $20 \mathrm{~min}$ at $4^{\circ} \mathrm{C}$. $\mathrm{OHCs}$ were rinsed in cold PBS and lysed using M-Per buffer (Perbo Pierce Laboratories), supplemented with Complete for 20 $\min$ at $4^{\circ} \mathrm{C}$. Extracts were centrifuged $(10000 \mathrm{x} \mathrm{g}, 10 \mathrm{~min})$ and the supernatant was transferred into a new tube and stored at $-80^{\circ} \mathrm{C}$ until use. Protein concentration was determined using Bradford reagent. $30 \mu \mathrm{g}$ total protein were loaded per lane and separated under denaturing conditions on a 12\% polyacrylamide gel. Proteins were transferred to nitrocellulose membranes by electroblotting. Membranes were stained with PonceauS to check efficiency of the transfer. For Western Blotting the membranes were blocked with $2 \%$ BSA in TBST for 1 hour at $37^{\circ} \mathrm{C}$. After washing in TBST, membranes were incubated in a TBST-solution containing the 1st antibodies (rabbit anti- BiP/Grp78 (Abcam, 1:1000); murine GAPDH (Abcam, 1:1000); rabbit anti-Grp94 (Abcam, 1:1000); rabbit anti- $\beta$-actin (Abcam, 1:500); mouse anti-GAPDH [6c5] (Abcam, 1:10000)). After washing and incubation with the corresponding HRP linked 2nd antibody, the membranes were developed using ECL reagent (Perbo Pierce Laboratories). Chemiluminescence was 
visualized by using an ECL hypersensitive film (Amersham Biosciences). Densitometric evaluation was performed using the program Kodak Digital Science 1D.

\subsection{Semiquantitative RT-PCR}

For semiquantitative RT-PCR, RNA was isolated using the Qiagen RNA isolation kit. Reverse transcription was performed using oligo-dT primer and superscript reverse transcriptase. The following primers were used for amplification: mu18S: 5'- TGATTAAGTCCCTGCCCTTT-3' and CAAGTTCGACCGTCTTCTCA-3'; muXbp1: 5'-CCTTGTGGTTGAGAACCAGG-3' and 5'-GAGGCTTGGTGTATACATGG-3', muBiP: 5'-GCACGTGTGTCGGCATCG-3' and 5'-GTCCAGCAATAGTGCCAGC-3'. For mu18S amplification, the template was diluted $1: 100$. The annealing temperature was $70^{\circ} \mathrm{C}$ for mu $18 \mathrm{~S}$ and $58^{\circ} \mathrm{C}$ for muXbp 1 and muBiP. ImageJ program was used for quantification (https://imagej.nih.gov/ij/docs/index.html).

\section{Results}

In order to investigate the molecular mechanisms that result in loss of stressed neurons OHCs were treated with tunicamycin $(300 \mathrm{ng} / \mathrm{ml}$ ) for 48 hours and then transferred to control medium for up to 2 days according to the protocol established before [22]. These experimental conditions allow for monitoring the morphology and metabolism of neuronal and glial cells in intact hippocampal tissue. Application of higher tunicamycin concentrations destroyed the integrity of the tissue.

\subsection{Different stressors elicit specific stress patterns}

In order to monitor the cellular stress response in OHCs, we performed BiP-specific immunohistochemical stainings of control and tunicamycin treated OHCs. In control sections NeuN positive neurons in CA1 express less BiP fluorescence signal than neurons in CA2/CA3 (compare overlays of green NeuN and red BiP signals). Lowest BiP levels prevail in cells that form the dentate gyrus. A faint BiP immunofluorescence is seen in the hippocampal laminae that contain the dendrites of pyramidal neurons and astrocytes (Fig. 1A). Tunicamycin treatment led to drastically increased BiP amounts especially in non-neuronal cells (Fig. 1B). GFAP immunostaining proved these cells to be astrocytes (Fig. 2). BiP levels remained low in all NeuN positive neurons of all hippocampal subfields. In the dentate gyrus, BiP levels remain as low as in control sections. SubAB treatment of OHCs only allowed examination of the neuronal and glial metabolism in intact tissue when very low concentrations of SubAB (10 ng/ml) were applied. Higher amounts (50 $\mathrm{ng} / \mathrm{ml}$ ) resulted in a severe damage of the cultured slices and did not allow prolonged cultivation and subsequent immunohistochemical analysis. After SubAB treatment (48 hours incubation at a concentration of $10 \mathrm{ng} / \mathrm{ml}$ and $2 \mathrm{days}$ recovery) no increases in BiP levels were detectable, neither in neurons nor in glial cells (Fig. 1C).

These results show that astrocytes respond strongly to accumulated protein in the ER by upregulating BiP levels; little response is monitored in neuronal cells of all hippocampal regions. SubAB, which cleaves BiP in the absence of misfolded protein, does not elicit a glial response.

\subsection{Activation of caspase-3 in control and tunicamycin treated OHCs}

In order to visualize cells undergoing apoptosis we investigated control and toxin-treated OHCs immunohistochemically using caspase-3 staining. After tunicamycin treatment we observed neuronal death in CA1 and even more severe in the dentate gyrus, while SubAB treatment obviously did not induce any significant apoptotic effects that could be attributed to a specific cell population (Fig. 3). These results indicate that stressed hippocampal neurons with low BiP levels (CA1 pyramids and dentate granule cells) are highly vulnerable to ER stress elicited by tunicamycin.

The data show that different toxins elicit different cellular responses. Tunicamycin, which inhibits glycosylation of proteins and leads to accumulation of misfolded proteins, induces the upregulation of BiP especially in the glial cell population and apoptosis in neurons in dentate gyrus and CA1, hippocampal regions which express low levels of BiP. Low concentrations of SubAB, which cleave BiP thereby leading to BiP depletion in the absence of protein aggregate formation, induce a general stress response in all cells in the OHCs without the activation of an apoptotic pathway in a specific hippocampal region.

\subsection{Analyses of the stress response in tunicamycin and SubAB treated OHCs}

Since in SubAB treated OHCs, neither increased BiP expression nor significant apoptosis were observed, we tested whether SubAB was principally taken up by the cells. We therefore analysed SubAB-specific cleavage of BiP by Western blot analyses in whole tissue lysates of OHCs. SubAB induced BiP cleavage in a dose-dependent manner (Fig. 4). Full length BiP decreased while the $28 \mathrm{kDa}$-terminal fragment accumulated. We concluded that SubAB was taken up by 
the cells in the OHCs. Further, we analysed whether cleavage of BiP by SubAB induced splicing of Xbp1 and transcriptional upregulation of BiP. OHCs were incubated with various SubAB concentrations $(10 \mathrm{ng} / \mathrm{ml}, 20 \mathrm{ng} / \mathrm{ml}, 50$ $\mathrm{ng} / \mathrm{ml})$ and tunicamycin (300 $\mathrm{ng} / \mathrm{ml})$ for the time period indicated. Subsequently, lysates were analysed for their RNA levels of Xbp1 (unspliced and spliced) and BiP (Fig. 5). SubAB and tunicamycin induced a similar strong stress response resulting in a 9-to-10-fold transcriptional upregulation of $\mathrm{BiP} 48 \mathrm{~h}$ after toxin application. As visualized by the appearance of the $\mathrm{Xbp} 1$ double band, $\mathrm{Xbp} 1$ splicing occurred after $6 \mathrm{~h}$ irrespective of the toxin or concentration applied to the OHCs. These results show that both cleavage of BiP by SubAB as well as accumulation of misfolded proteins due to tunicamycin application elicit a comparable stress response that induces Xbp1 splicing as well as transcriptional upregulation of BiP.

\subsection{Response of cultured glial and neuronal cells to tunicamycin-induced ER stress}

As shown by immunohistochemical stainings, tunicamycin induces a strong astrocyte response. Accordingly, we asked whether the tunicamycin-mediated upregulation of BiP in glial cells is cell type specific or due to a metabolic crosstalk between neurons and glial cells. We hypothesized that BiP upregulation in glial cells could be i) based on neuronal stress, which elicits the glial stress response, or ii) due to an earlier onset and more pronounced stress response of glial cells. To elucidate the sensitivity of neuronal and glial cells to either accumulated proteins, disturbances of the ionic homeostasis or BiP depletion, we isolated and cultured primary neuronal and glial cells to test their stress responses individually. When checking the purity of the primary cultures we observed that the neuronal cells were always contaminated by GFAP-positive glial cells and glial cells proliferated rapidly. Instead of primary neurons we therefore used the second best possibility, a neuronal cell line (N2a), for subsequent experiments. For comparison of the dynamics of neuronal and glial stress responses all stress experiments were strictly performed in parallel to ensure that toxin concentrations and time points of cell harvest were absolutely identical for both cultures. N2a and glial cells were treated with tunicamycin, thapsigargin (an ER stressor that disturbs cellular calcium homeostasis due to inhibition of the Ca2+-dependent ATPase activity), or SubAB for the whole time (up to 24 hours) indicated (Fig. 6). When treated with tunicamycin or thapsigargin, the stress response - as determined by upregulation of BiP and Grp94 protein levels - was delayed in neuronal cells, which means that astroglial cells respond faster towards ER stress by upregulating ERresident chaperones. This delayed neuronal response could be either due to a reduced sensitivity of neuronal cells or due to a delayed toxin uptake. Toxin uptake can be estimated in the SubAB treated cultures by considering the kinetics of the appearance of the BiP cleavage product, the $28 \mathrm{kDa} \mathrm{C}$-terminal fragment that can be visualized by Western blot as soon as cleavage by SubAB occurs. This cleaved $28 \mathrm{kDa}$ band can be detected earlier in the glial cell culture than in the neuronal population indicating that SubAB is taken up faster by the astroglial cells (Fig. 6). Interestingly, SubAB treatment strikingly reduced actin levels in neuronal cells, but not in astroglial cells (Fig. 6). Due to the use of a neuronal cell line versus primary astroglial cells and the fact that cultured cells have a rather different physiology compared to those in vivo these experimental results have to be considered with caution. Nevertheless, as the neuronal cell line is less robust than the primary astroglial cells we deduce the faster stress response in glial cells correlates with a higher stress resistance.

\subsection{Are neurons in the dentate gyrus protected by prestress?}

Tunicamycin as well as SubAB treatment induce the UPR, specifically in glial cells. The underlying mechanisms are different though: while tunicamycin induces the accumulation of misfolded proteins, SubAB cleaves BiP, both resulting in subsequent upregulation of BiP to rebalance the appropriate protein to chaperone ratio. We hypothesized that prestressing of cells by low dosages of SubAB might induce chaperone synthesis, the elevated levels of which should protect hippocampal neurons in CA1 and dentate gyrus from tunicamycin-induced apoptosis. To test this, we incubated the OHCs for 48 hours with SubAB and transferred them to tunicamycin-containing medium for another 48 hours. Afterwards, we performed immunohistochemical stainings to monitor the regional localization and amount of BiP and cleaved caspase-3 (Fig. 7). Preincubation with SubAB (10 ng/ml) showed no protective effect to subsequent incubation with tunicamycin (300 ng/ml) (data not shown). We, therefore, stepwise reduced the SubAB concentration and found that preconditioning with an extremely low dosage of $1 \mathrm{ng} / \mathrm{ml}$ SubAB in fact had a protective effect: Subsequent tunicamycin treatment did not reveal cleaved caspase- 3 positive cells, suggesting preserved neuronal viability. Based on our experimental data we conclude that a pre-stress has a protective potential for hippocampal neurons, provided that the pre-stress remains beyond a critical, rather low level.

\section{Discussion}

\subsection{CA1 and dentate gyrus are the most vulnerable regions in the hippocampus}

When treating OHCs with tunicamycin, we observed a drastic increase in cleaved caspase- 3 both, in the dentate gyrus and in the CA1 region, indicating that the neurons in these regions are most vulnerable to stress. The dentate gyrus is 
known to be a highly vulnerable region within the hippocampal formation. Amyloid plaques and neurofibrillary tangles are deposited in the dentate gyrus at an early stage of $\mathrm{AD}[23,24]$ and the observed neuronal loss is associated with deficits in memory processing and sensory inputs [25]. A progressive and dramatic loss of granular neurons and astrogliosis also occurred in the dentate gyrus of the hippocampus in a mouse model with an inducible TDP-43 pathology [26]. TDP-43 pathology occurs in frontotemporal dementia and in about 30\% of AD and is characterized by aberrant accumulation of insoluble inclusions in the cytosol of neurons and glia. The comparable neuronal loss in AD and tunicamycin-treated OHCs supports the validity of this experimental model to investigate the early metabolic patterns and cellular stress responses that eventually lead to neuronal loss.

\subsection{Upregulation of BiP in astroglia cells}

By means of this experimental model, we observed a massive increase in BiP levels in astrocytes upon tunicamycin treatment. In order to investigate whether this increase is due to a higher sensitivity of glial cells to stress resulting in a stronger and/or faster ER stress response, we compared this parameter in cultured neuronal and glial cells. Interestingly, astroglial cells respond faster and stronger to ER stressors such as tunicamycin or thapsigargin. Thus astroglia cells appear to counteract the imposed metabolic changes to maintain the ionic and protein balance of the extracellular micromilieu. A specific stress response of astroglial cells in the hippocampus was also reported in rat brains after acute intracerebroventricular injection of $\beta$-amyloid oligomers, which induced overexpression of GFAP and GRP78 [27].

ER stress sensing by monitoring the kinetics of spliced Xbp1-mRNA revealed similar onsets in neurons and astroglia, indicating that ER stress and subsequent activation of the IRE/Xbp1 branch is comparable in both cell types. Irrespective of this, the corresponding neuronal response is delayed and possibly not as pronounced as the astroglial response. In this context it is of interest that astrocytes have a cell specific stress transducer OASIS (old astrocyte specifically induced substance), which is located in the ER membrane. OASIS has been shown to be strongly upregulated in ER stressed hippocampal astrocytes [28]. It has a similar structure and function as ATF6 but might transmit ER stress and subsequent stress response more efficiently to the nucleus [29]. The sanity and intactness of the astroglial metabolism is of decisive value, since the neuronal metabolism, its function and survival is guarded and controlled by astrocytes [30-32]. Astrocytes are known to be more resilient than neurons and they play a fundamental role in the pathogenesis of brain injury associated with neuronal death. Therefore they are more and more in focus of research to develop therapeutic treatments to ameliorate the outcome of neurodegenerative diseases [32, 33].

\subsection{Tunicamycin and SubAB elicit different stress responses}

It is still unclear whether the UPR transducers, IRE1, PERK and ATF6 are activated by dissociation of BiP from their luminal domain or whether their activation is initiated by the increasing amounts of misfolded protein in the ER [34]. According to our results, both the accumulation of misfolded proteins (by tunicamycin treatment) or inactivation of BiP (by SubAB cleavage) result in splicing of Xbp1 in a comparable way, indicating that the IRE1 - Xbp1 branch of the UPR is activated by BiP cleavage or its dissociation from the IRE1 transmembrane receptor. Also the temporal profile of transcriptional upregulation of Grp94 is comparable in both experimental conditions, indicating that there is no specific UPR stress response that is activated by increasing amounts of accumulated protein per se - at least not in cultured neuronal and astroglial cells.

Yet in OHCs different stress patterns are observed: while tunicamycin treatment activates astroglial stress response (as observed by upregulated BiP levels), SubAB treatment did not elicit an astrocyte-confined response, but rather resulted in a general upregulation of BiP in neurons and astrocytes. Taking into account that in cell culture, astroglial cells respond earlier to ER stress than neurons irrespective of the stressor used, the strong astroglial response in OHCs upon tunicamycin treatment could be cell type specific and even amplified due to intact, intercellular communication and network in this experimental model. On the other hand, the general loss of BiP - induced by SubAB - is not counterbalanced by glial cells, and could reflect the metabolic pattern in aging brains with generally lower chaperone levels and dispersed degeneration of neurons and astrocytes.

Due to the different metabolic patterns observed in tunicamycin- and SubAB-treated OHCs we conclude that astrocytes have the ability to sensitively register and respond to the accumulation of misfolded proteins. Since there is no difference in the temporal profile of Xbp1 splicing we believe that another branch of the UPR is activated by misfolded proteins in the ER, possibly the ATF6/OASIS branch, which is known to enforce the UPR response and to synergistically activate the transcription of $\mathrm{BiP}$, other chaperones or components of the ER- associated degradation machinery. 


\subsection{Preconditioning}

In order to rebalance metabolism in aging or diseased brains, one might assume that elevation of the endogenous BiP pool could ameliorate survival of hippocampal neurons in CA1 and dentate gyrus. However, elevation of the endogenous $\mathrm{BiP}$ pool is not easy to achieve since the cells counter-regulate their BiP levels at posttranscriptional level when BiP is overexpressed in unstressed cells [35]. We, therefore, elevated endogenous chaperone levels by application of SubAB and can demonstrate that this "preconditioning of the OHCs" with low doses of SubAB in fact protects neurons against subsequent tunicamycin-elicited stress and subsequent cell death. Successful preconditioning has been reported before in a mouse model of AD by repeated cold exposure [36]. Preconditioning is for long known to protect against ischemic injury. As in our experimental setting, preconditioning needs to be achieved by a non-injurious preconditioning stimulus that increases the resistance of the brain tissue to subsequent prolonged anoxia [37]. Stress tolerance has also been reported for other stimuli such as hyperoxia, oxidative stress, hyperthermia or heat shock, showing that one stressor can promote cross-tolerance to another [37]. This possibly offers a novel approach to develop chemical compounds that precondition the brain tissue so that it is more resistant to subsequent stressors such as imbalances in the protein to chaperone ratio. The challenge will be to develop stimuli that are potent enough to evoke a protective response yet not too strong to elicit a harmful response.

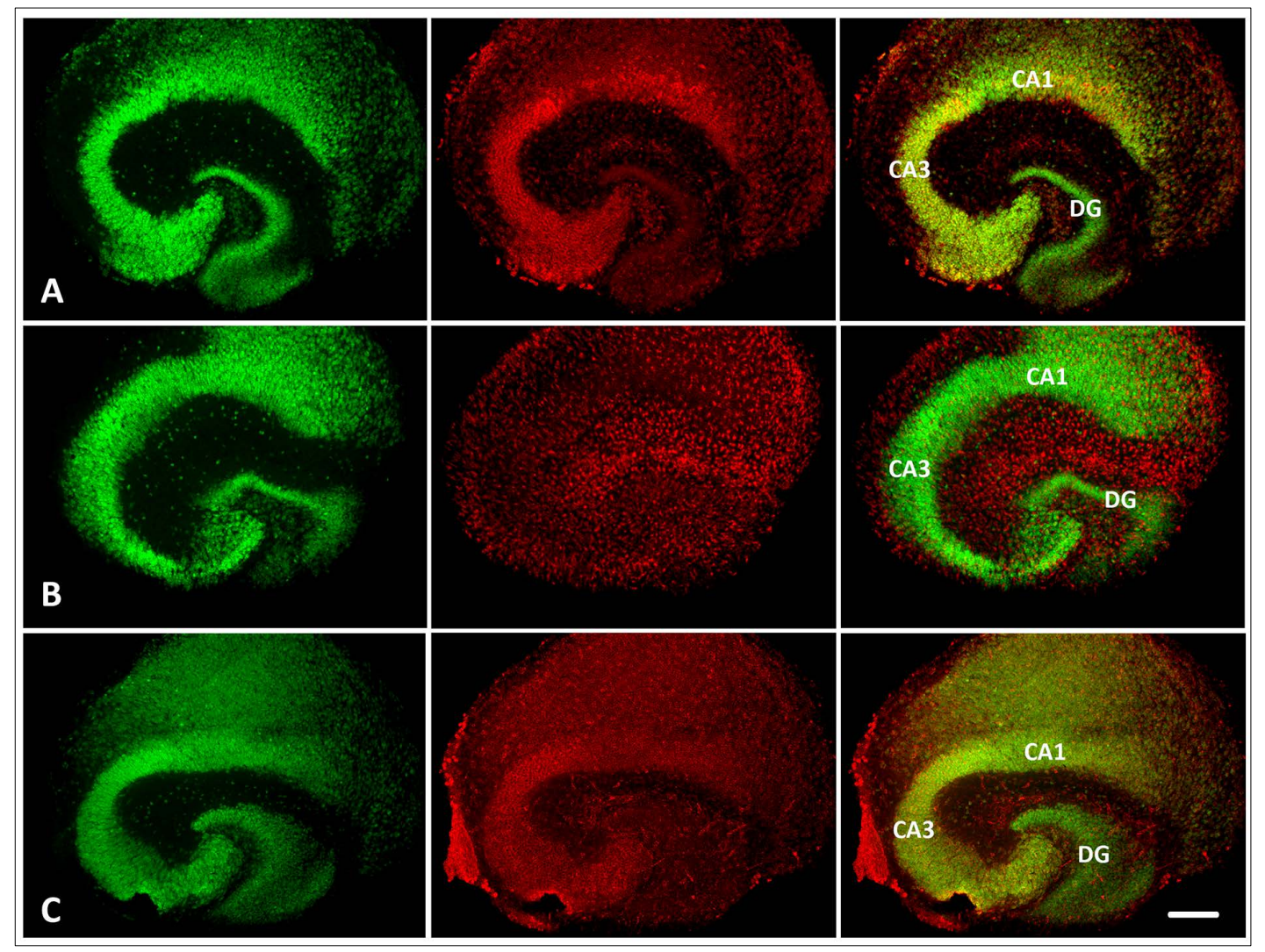

Figure 1 Stress response of neuronal cells and astrocytes in OHCs. OHCs were cultured in control medium for 3 days and then for another $48 \mathrm{~h}$ either in control medium (A), in tunicamycin-containing medium (300 $\mathrm{ng} / \mathrm{ml})$ (B), or in SubAB- containing medium (10 ng/ml) (C). Medium was exchanged and OHCs were allowed to recover for another $48 \mathrm{~h}$ in control medium prior to their fixation. OHCs were sliced and stained immunohistochemically with the neuronal marker NeuN (green) and BiP (red). Typical protein patterns are shown from three independent experimental series. To avoid overexposure of the bright glial BiP signal (B), exposure time is kept short and BiP expression in hippocampal neurons is not visible. Scale bar $200 \mu \mathrm{m}$. 


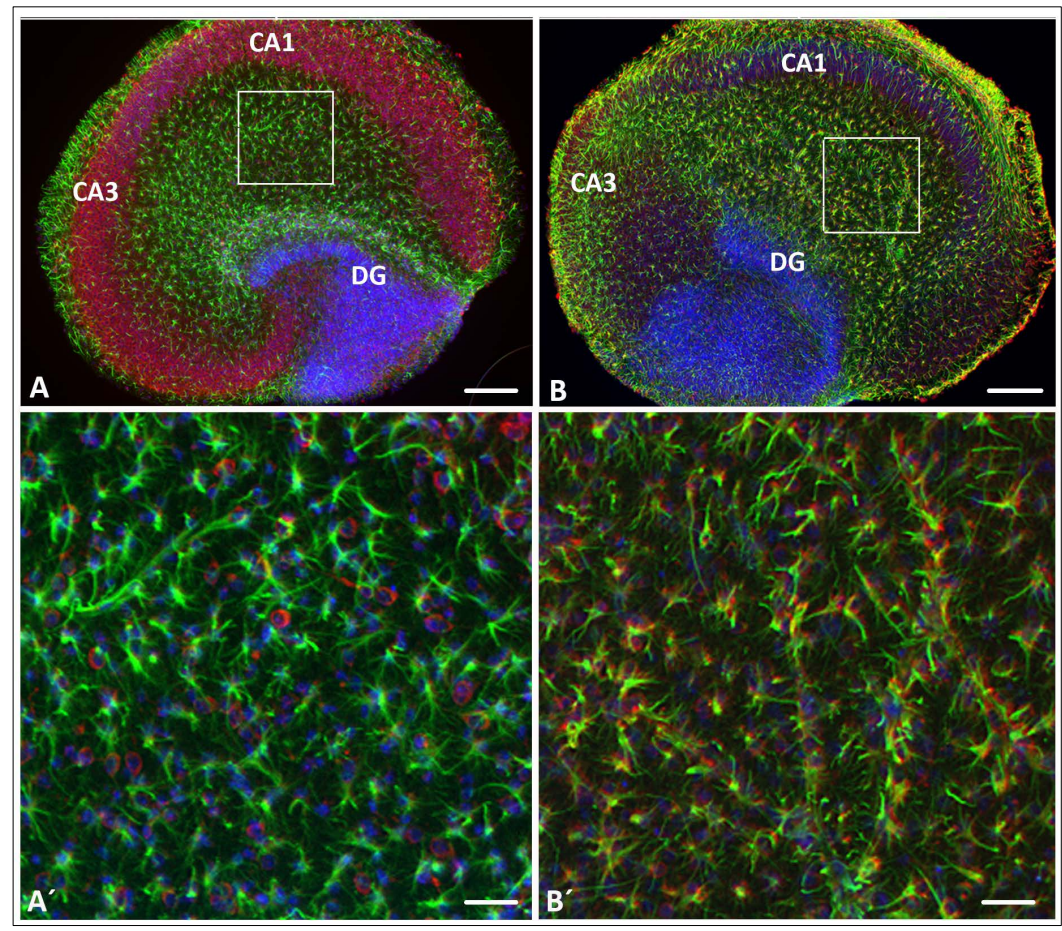

Figure 2 Elevated BiP levels in tunicamycin treated GFAP-positive astrocytes. OHCs were cultured in control medium (A) or treated with tunicamycin (300 ng/ml) (B). Immunostaining was performed for BiP (red) and the astrocyte marker GFAP (green). Nuclei are stained with DAPI (blue). A', B') Enlargement of the rectangle- marked region in A) and B). To avoid overexposure of the bright glial BiP signal (B, $\mathrm{B}^{\prime}$ ), exposure time is kept short and BiP expression in hippocampal neurons is not visible. A representative staining pattern out of two experimental series is shown. Note the BiP pattern in tunicamycin- treated OHCs is largely due to elevated BiP levels in astrocytes. A, B) scale bar $200 \mu \mathrm{m}$; $A^{\prime}, B^{\prime}$ ) scale bar $37 \mu \mathrm{m}$.

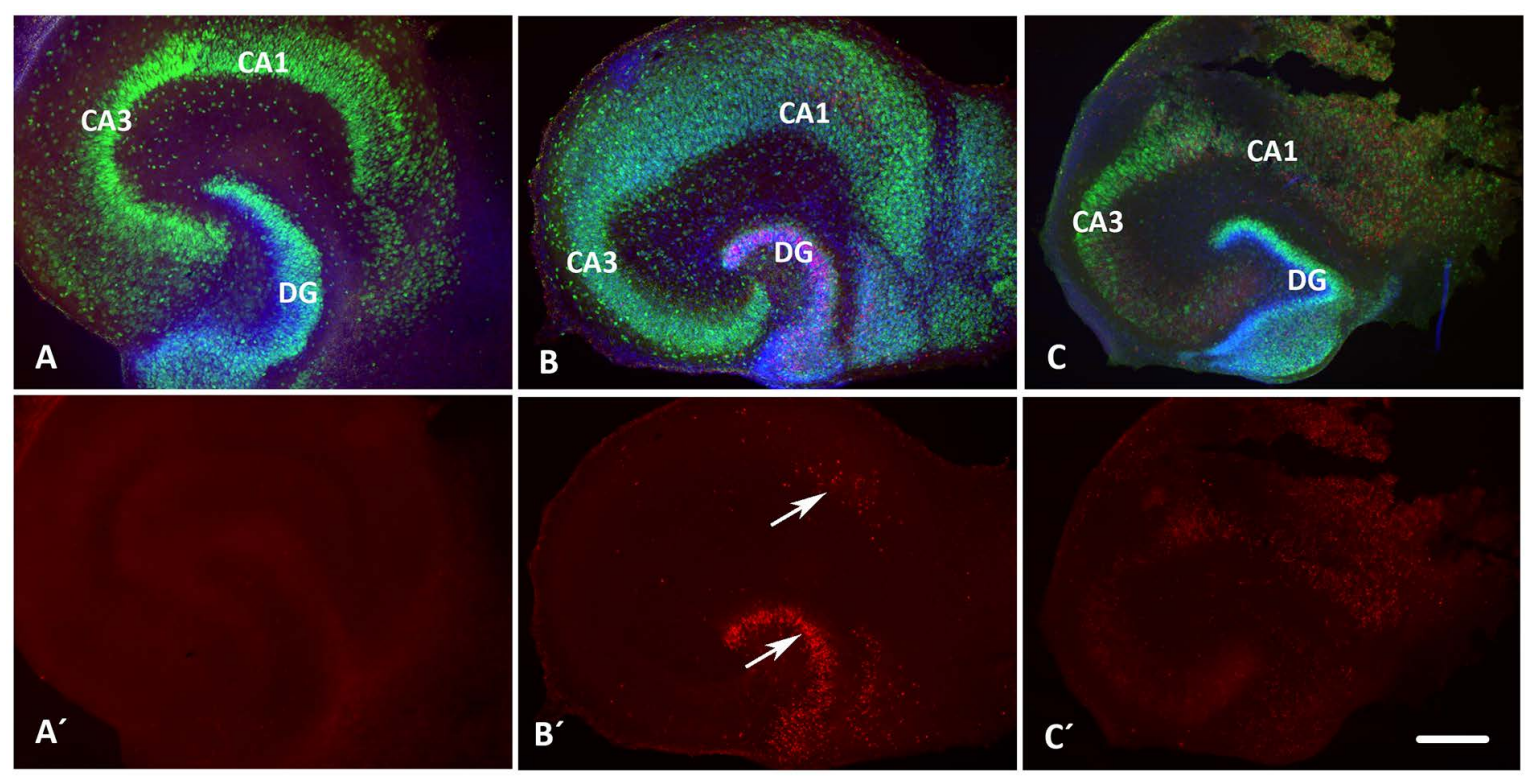

Figure 3 Cleaved caspase-3 immunostaining to monitor stress-induced apoptosis. OHCs were cultured in control medium for 5 days and then either for another $48 \mathrm{~h}$ in control $\left(\mathrm{n}=3 ; \mathrm{A}, \mathrm{A}^{\prime}\right)$, in tunicamycin- $(300 \mathrm{ng} / \mathrm{ml})\left(\mathrm{n}=3\right.$; $\left.\mathrm{B}, \mathrm{B}^{\prime}\right)$ or SubAB- containing medium $(\mathrm{n}=3 ; 10 \mathrm{ng} / \mathrm{ml})\left(\mathrm{C}, \mathrm{C}^{\prime}\right)$ prior to fixation. The staining pattern for cleaved caspase-3 visualized apoptotic cells in CA1 and dentate gyrus in tunicamycin treated OHCs (white arrows). Experiments were performed three times with OHCs prepared from different mouse brains. Scale bar $200 \mu \mathrm{m}$. 


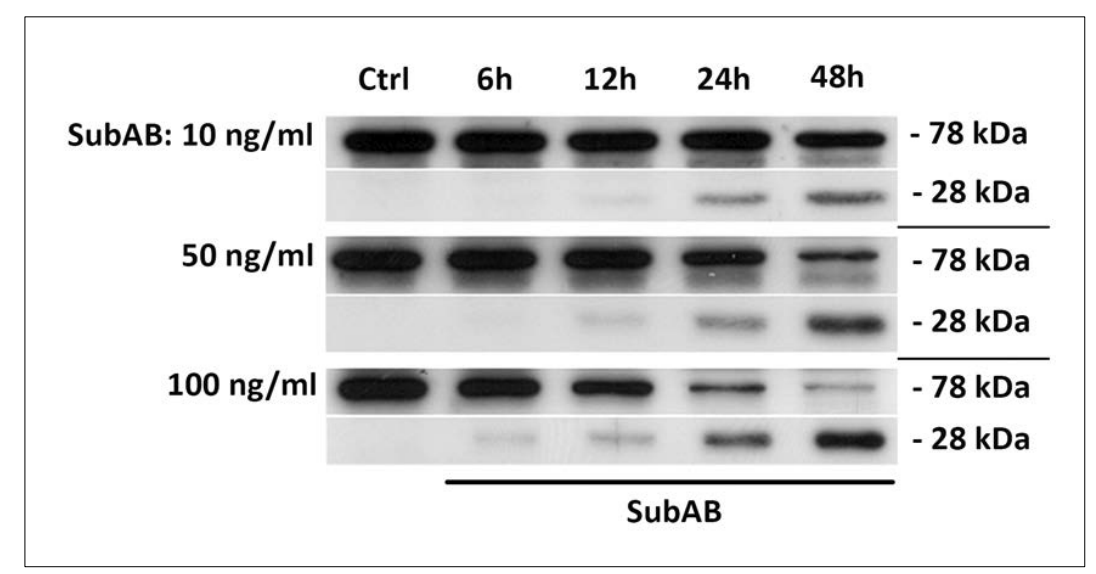

Figure 4 Verification of SubAB activity at the low concentrations used to stress OHCs. OHCs incubated with SubAB were lysed and subsequently analysed by Western Blot. The $28 \mathrm{kDa}$ C-terminal fragment of BiP is generated by SubAB cleavage.

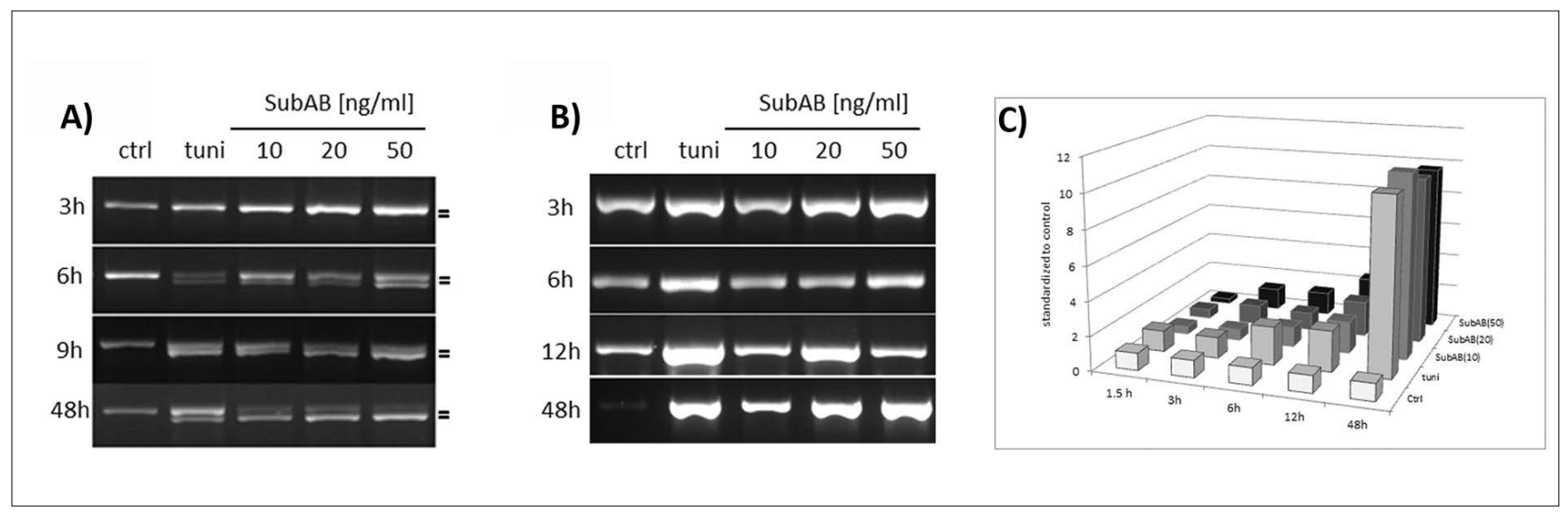

Figure 5 Comparable kinetics of the tunicamycin- and SubAB stress-induced signalling cascades. Comparison of the effectiveness of the toxins to splice $\mathrm{Xbpl}(\mathrm{A})$ and to upregulate $\mathrm{BiP}(\mathrm{B})$. OHCs were treated with tunicamycin (tuni; 300 $\mathrm{ng} / \mathrm{ml})$ or SubAB at the concentrations indicated $(10 \mathrm{ng} / \mathrm{ml}, 20 \mathrm{ng} / \mathrm{ml}$, and $50 \mathrm{ng} / \mathrm{ml})$. Quantification of BiP levels was performed using lmageJ (C). BiP values were normalized to $18 \mathrm{~S}$ (which served as internal sample control) and standardized to control values (Ctrl). 


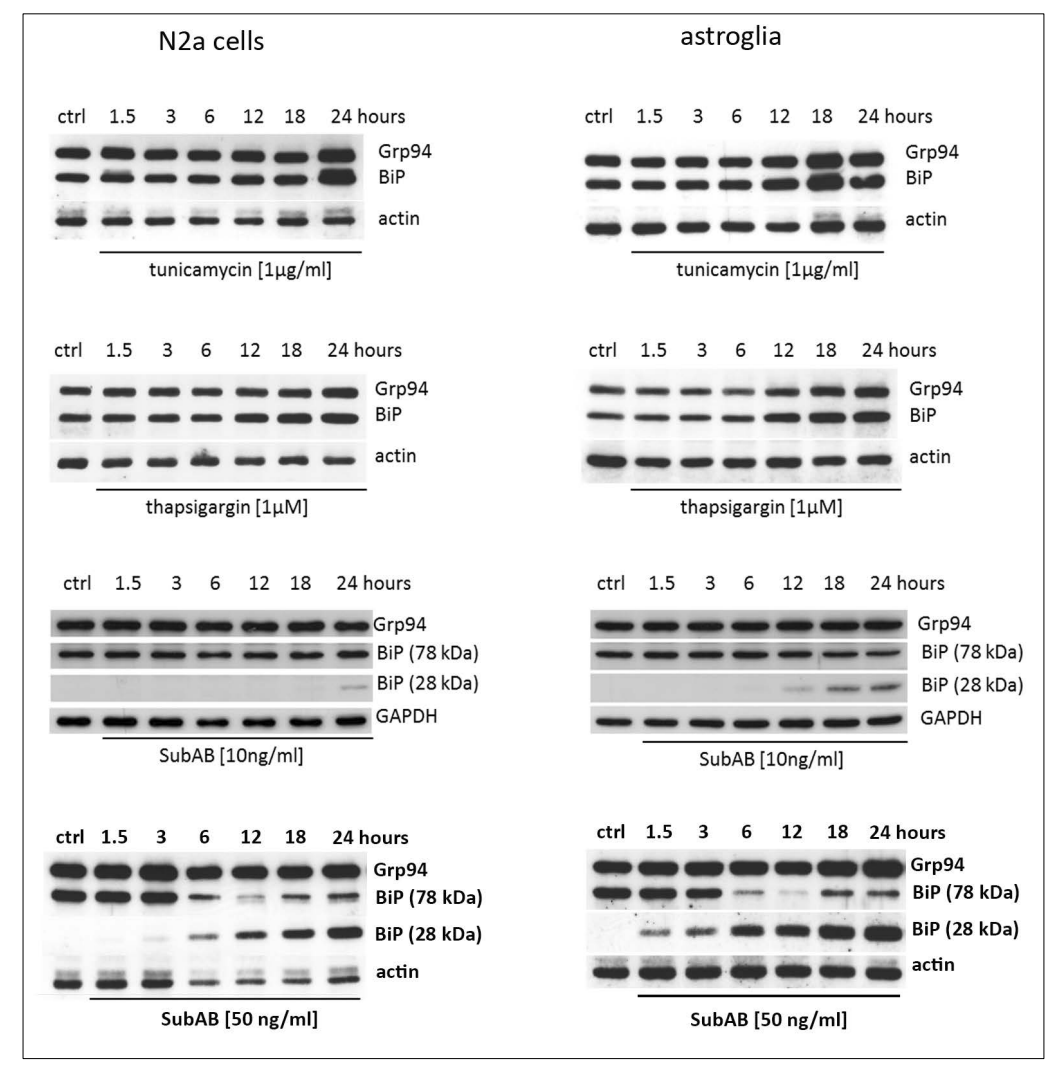

Figure 6 Comparison of the stress-induced upregulation of BiP and Grp94 protein levels in cultured astroglia and neuronal cells (N2a). Astroglial and neuronal cells were incubated with tunicamycin, thapsigargin or SubAB for the time indicated. Cells were lysed and BiP and Grp94 levels were visualized by Western Blot. The $28 \mathrm{kDa}$ BiP band corresponds to the C-terminal cleavage fragment generated by SubAB treatment. GAPDH or actin staining served as loading control.
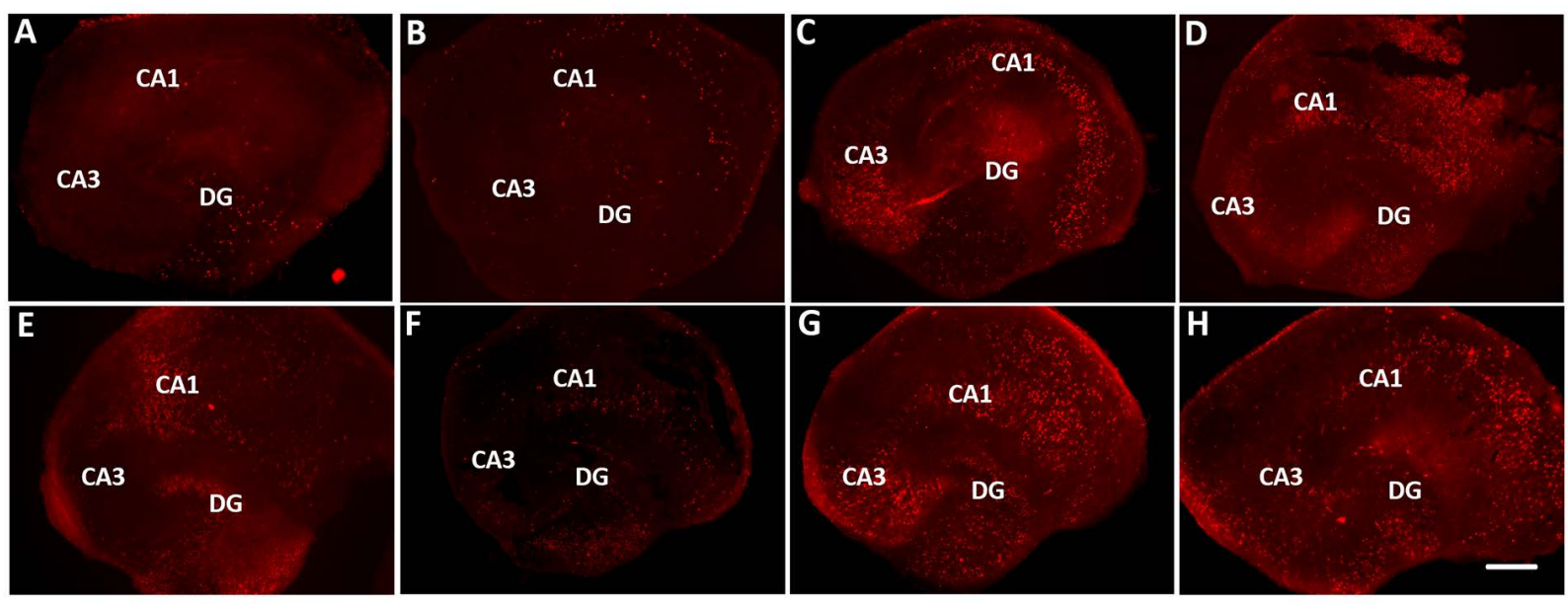

Figure 7 Preconditioning of OHCs with low doses of SubAB promotes survival of hippocampal neurons in CA1 and DG. OHCs were cultured in control medium for 3 days. At day 3 OHCs were further cultured for another $48 \mathrm{~h}$, either in control medium (A), or SubAB-containing medium: $1 \mathrm{ng} / \mathrm{ml}$ (B), $2.5 \mathrm{ng} / \mathrm{ml}$ (C) or $5 \mathrm{ng} / \mathrm{ml}$ (D). At day 5 medium was exchanged to control medium and OHCs were fixed at day 7, sectioned and stained for cleaved caspase-3. (E) At day 5 OHCs were incubated in tunicamycin $(300 \mathrm{ng} / \mathrm{ml})$ for 48 hours and fixed at day 7. Preconditioning of OHCs (F-H): At day 3, OHCs were preincubated with SubAB $1 \mathrm{ng} / \mathrm{ml}(\mathrm{F}), 2.5 \mathrm{ng} / \mathrm{ml}(\mathrm{G})$ or $5 \mathrm{ng} / \mathrm{ml}(\mathrm{H})$ for $48 \mathrm{~h}$, followed by incubation in tunicamycin-containing medium $(300 \mathrm{ng} / \mathrm{ml}$, day 5-7) for another 48 prior to fixation, sectioning and staining with cleaved caspase-3. All experiments were done three times with OHCs prepared from different mouse brains. Scale bar $200 \mu \mathrm{m}$. 


\section{Conclusion}

In aged brains, chaperone levels decrease while mutational rates increase, an imbalance that leads to the accumulation of misfolded proteins that form aggregates in diseased brains resulting in cellular stress and subsequent neurodegeneration. Most vulnerable to ER-stress is the hippocampus, the central region for learning ability and memory. We show that the hippocampal CA1 region and the dentate gyrus are most vulnerable to tunicamycin-induced ER-stress. Furthermore, a strong astroglial response is observed possibly promoting survival of CA3 neurons. In our experimental system, mild prestressing of OHCs protected hippocampal neurons and promoted their survival. Elaboration of prestressing conditions revealed that prestress is only protective when remaining below a low and rather critical levels. To protect aging brains against neurodegeneration further conditions need to be elaborated that allow appropriate prestressing of neuronal cells and which support astroglial stress response.

\section{Compliance with ethical standards}

\section{Acknowledgments}

We thank Simone Zenker (Institute of Anatomy and Cell Biology, Freiburg) and Bettina Kruck (UKE, Hamburg) for their excellent technical assistance.

\section{Disclosure of conflict of interest}

There is no conflict of interest.

\section{References}

[1] Chadwick SR, Lajoie P. Endoplasmic Reticulum Stress Coping Mechanisms and Lifespan Regulation in Health and Diseases. Front Cell Dev Biol. 2019; 7: 84.

[2] Adams CJ, Kopp MC, Larburu N, Nowak PR, Ali MMU. Structure and Molecular Mechanism of ER Stress Signaling by the Unfolded Protein Response Signal Activator IRE1. Front Mol Biosci. 2019; 6(6): 11.

[3] Harding HP, Zhang Y, Ron D. Protein translation and folding are coupled by an endoplasmic-reticulum-resident kinase. Nature. 1999; 397(6716): 271-4.

[4] Hollien J, Weissman JS. Decay of endoplasmic reticulum-localized mRNAs during the unfolded protein response. Science. 2006; 313(5783): 104-7.

[5] Wang Y, Shen J, Arenzana N, Tirasophon W, Kaufman RJ, Prywes R. Activation of ATF6 and an ATF6 DNA binding site by the endoplasmic reticulum stress response. The Journal of biological chemistry. 2000; 275(35): 2701320 .

[6] Zhang Y, Liu R, Ni M, Gill P, Lee AS. Cell surface relocalization of the endoplasmic reticulumchaperone and unfolded protein response regulator GRP78/BiP. The Journal of biological chemistry. 2010; 285(20): 15065-75.

[7] Ni M, Zhang Y, Lee AS. Beyond the endoplasmic reticulum: atypical GRP78 in cell viability, signalling and therapeutic targeting. Biochem J. 2011; 434(2): 181-8.

[8] Campanella C, Pace A, Caruso Bavisotto C, Marzullo P, Marino Gammazza A, Buscemi S, et al. Heat Shock Proteins in Alzheimer's Disease: Role and Targeting. International journal of molecular sciences. 2018; 19(9).

[9] Pradeepkiran JA, Reddy PH. Structure Based Design and Molecular Docking Studies for Phosphorylated Tau Inhibitors in Alzheimer's Disease. Cells. 2019; 8(3).

[10] Goedert M, Jakes R, Spillantini MG, Crowther RA, Cohen P, Vanmechelen E, et al. Tau protein in Alzheimer's disease. Biochem Soc Trans. 1995; 23(1): 80-5.

[11] Steiner H, Fukumori A, Tagami S, Okochi M. Making the final cut: pathogenic amyloid-beta peptide generation by gamma-secretase. Cell Stress. 2018; 2(11): 292-310.

[12] Kayed R, Lasagna-Reeves CA. Molecular mechanisms of amyloid oligomers toxicity. J Alzheimers 4 Dis. $2013 ; 33$ Suppl 1: S67-78.

[13] Posimo JM, Weilnau JN, Gleixner AM, Broeren MT, Weiland NL, Brodsky JL, et al. Heat shock protein defenses in the neocortex and allocortex of the telencephalon. Neurobiol Aging. 2015; 36(5): 1924-37. 
[14] Hoozemans JJ, Veerhuis R, Van Haastert ES, Rozemuller JM, Baas F, Eikelenboom P, et al. The unfolded protein response is activated in Alzheimer's disease. Acta Neuropathol. 2005; 110(2): 165-72.

[15] Hoozemans JJ, van Haastert ES, Nijholt DA, Rozemuller AJ, Eikelenboom P, Scheper W. The unfolded protein response is activated in pretangle neurons in Alzheimer's disease hippocampus. The American journal of pathology. 2009; 174(4): 1241-51.

[16] Marcora MS, Belfiori-Carrasco LF, Bocai NI, Morelli L, Castano EM. Amyloid-beta42 clearance and neuroprotection mediated by X-box binding protein 1 signaling decline with aging in the Drosophila brain. Neurobiol Aging. 2017; 60: 57-70.

[17] Cisse M, Duplan E, Lorivel T, Dunys J, Bauer C, Meckler X, et al. The transcription factor XBP1s restores hippocampal synaptic plasticity and memory by control of the Kalirin-7 pathway in Alzheimer model. Mol Psychiatry. 2017; 22(11): 1562-75.

[18] Duran-Aniotz C, Cornejo VH, Espinoza S, Ardiles AO, Medinas DB, Salazar C, et al. IRE1 signaling exacerbates Alzheimer's disease pathogenesis. Acta Neuropathol. 2017; 134(3): 489-506.

[19] Paton AW, Beddoe T, Thorpe CM, Whisstock JC, Wilce MC, Rossjohn J, et al. AB5 subtilase cytotoxin inactivates the endoplasmic reticulum chaperone BiP. Nature. 2006; 443(7111): 548-52.

[20] Wu YJ, Schulz H, Lin CC, Saar K, Patone G, Fischer H, et al. Borna disease virus-induced neuronal degeneration dependent on host genetic background and prevented by soluble factors. Proceedings of the National Academy of Sciences of the United States of America. 2013; 110(5): 1899-904.

[21] Zhou X, Spittau B, Krieglstein K. TGFbeta signalling plays an important role in IL4-induced alternative activation of microglia. J Neuroinflammation. 2012; 9: 210

[22] Lacour P, Heimrich B, Prols F. Induction of cellular stress and chaperone activation in organotypic slice cultures of hippocampus. J Neurosci Methods. 2007; 166(1): 24-31.

[23] Braak H, Braak E. Neuropathological stageing of Alzheimer-related changes. Acta Neuropathol. 1991; 82(4): 23959.

[24] Chen XQ, Mobley WC. Alzheimer Disease Pathogenesis: Insights From Molecular and CellularBiology Studies of Oligomeric Abeta and Tau Species. Front Neurosci. 2019; 13: 659.

[25] Kesner RP. An analysis of dentate gyrus function (an update). Behav Brain Res. 2018; 354: 84-91.

[26] Walker AK, Tripathy K, Restrepo CR, Ge G, Xu Y, Kwong LK, et al. An insoluble frontotemporal lobar degenerationassociated TDP-43 C-terminal fragment causes neurodegeneration and hippocampus pathology in transgenic mice. Hum Mol Genet. 2015; 24(25): 7241-54.

[27] Alberdi E, Wyssenbach A, Alberdi M, Sanchez-Gomez MV, Cavaliere F, Rodriguez JJ, et al. Ca(2+) -dependent endoplasmic reticulum stress correlates with astrogliosis in oligomeric amyloid beta-treated astrocytes and in a model of Alzheimer's disease. Aging Cell. 2013; 12(2): 292-302.

[28] Chihara K, Saito A, Murakami T, Hino S, Aoki Y, Sekiya H, et al. Increased vulnerability of hippocampal pyramidal neurons to the toxicity of kainic acid in OASIS-deficient mice. J Neurochem. 2009; 110(3): 956-65.

[29] Saito A, Hino S, Murakami T, Kondo S, Imaizumi K. A novel ER stress transducer, OASIS, expressed in astrocytes. Antioxid Redox Signal. 2007; 9(5): 563-71.

[30] Dossi E, Vasile F, Rouach N. Human astrocytes in the diseased brain. Brain Res Bull. 2018; 136: $139-56$.

[31] Vasile F, Dossi E, Rouach N. Human astrocytes: structure and functions in the healthy brain. Brain Struct Funct. 2017; 222(5): 2017-29.

[32] Barreto GE, Gonzalez J, Torres Y, Morales L. Astrocytic-neuronal crosstalk: implications forneuroprotection from brain injury. Neurosci Res. 2011; 71(2): 107-13.

[33] Benavides A, Pastor D, Santos P, Tranque P, Calvo S. CHOP plays a pivotal role in the astrocyte death induced by oxygen and glucose deprivation. Glia. 2005; 52(4): 261-75.

[34] Preissler S, Ron D. Early Events in the Endoplasmic Reticulum Unfolded Protein Response. Cold Spring Harb Perspect Biol. 2019; 11(4).

[35] Gulow K, Bienert D, Haas IG. BiP is feed-back regulated by control of protein translation efficiency. J Cell Sci. 2002; 115(Pt 11): 2443-52. 
World Journal of Biology Pharmacy and Health Sciences, 2021, 07(02), 019-031

[36] Tournissac M, Bourassa P, Martinez-Cano RD, Vu TM, Hebert SS, Planel E, et al. Repeated cold exposures protect a mouse model of Alzheimer's disease against cold-induced tau phosphorylation. Mol Metab. 2019; 22: 110-20.

[37] Gidday JM. Cerebral preconditioning and ischaemic tolerance. Nat Rev Neurosci. 2006; 7(6): 437-48. 